\title{
The effect of a red background on location backward masking by structure
}

\author{
JEFFREY S. BEDWELL \\ University of Central Florida, Orlando, Florida \\ JAMES M. BROWN \\ University of Georgia, Athens, Georgia \\ AND \\ Diana M. Orem \\ University of Central Florida, Orlando, Florida
}

\begin{abstract}
It has been established that diffuse red light partially suppresses the magnocellular (M) visual pathway. Previous research reported that metacontrast masking is reduced (improved accuracy) with a red background, consistent with a reduction in $\mathrm{M}$ pathway response from the mask. In contrast, a recent study used location backward masking by noise and found that accuracy decreased with a red background - theoretically due to suppression of the M pathway's initial localization of the target. The present study provides the first report examining the effect of red light on performance in a location backward masking by structure task. Results revealed a main effect of a red (as opposed to a green) background on reducing masking (improved accuracy) with a medium effect size $\left(\eta^{2}=.23\right)$. This effect was strongest at the 47 - and $60-\mathrm{msec}$ stimulus onset asynchronies. Results suggest that red light primarily decreases interference from the mask in location backward masking by structure.
\end{abstract}

The term visual backward masking refers to the phenomenon of reduced visibility of an initial "target" when that target is quickly followed by another stimulus, which is termed the mask. The variable interval between the onset of the target and the onset of the mask is termed the stimulus onset asynchrony (SOA). A predominant model of backward masking focuses on two unique but interactive physiological subsystems in the visual system (Livingstone \& Hubel, 1987). The transient system involves the magnocellular $(\mathrm{M})$ visual pathway and is primarily responsible for processing location information and movement. It is the relatively faster responding system with lower spatial resolution and greater contrast sensitivity. The sustained system involves the parvocellular $(\mathrm{P})$ visual pathway and is primarily responsible for processing detail and color. It is the relatively slower responding, higher spatial resolution system (Breitmeyer \& Ganz, 1976; Livingstone \& Hubel, 1987).

The $\mathrm{M}$ and $\mathrm{P}$ pathways interact during visual backward masking, influencing performance. One theory proposes that both target and mask elicit a fast $\mathrm{M}$ system response and a slower P system response (Purushothaman, Ögmen, $\&$ Bedell, 2000). This theory describes how target visibility is worse (i.e., masking is greater) when the slower $\mathrm{P}$ response to the target is disrupted by the $\mathrm{M}$ response to the mask. Target visibility increases as the SOA increases, because the $\mathrm{P}$ pathway is allowed more time to process the target before the interruption by the M pathway response to the mask occurs.

Single-cell recording research with monkeys has demonstrated that the activity in a portion of M pathway neurons is suppressed by diffuse red light (de Monasterio, 1978; Dreher, Fukada, \& Rodieck, 1976; Krüger, 1977; Livingstone \& Hubel, 1984; Wiesel \& Hubel, 1966). This effect has been demonstrated in humans on behavioral tasks, with reduced performance on M pathway-dependent tasks in the presence of a red background-including simple reaction time tasks to visual stimuli (Breitmeyer \& Breier, 1994), reading performance (Chase, Ashourzadeh, Kelly, Monfette, \& Kinsey, 2003), the perception of depth (Brown \& Koch, 2000), global motion (Chapman, Hoag, \& Giaschi, 2004), and stroboscopic motion (Breitmeyer \& Williams, 1990). This effect has also been suggested in an fMRI study in which cortical area V5 (MT+) —an area that receives substantial M pathway input-showed decreased activity in the right (as opposed to the left) hemisphere when observers viewed moving concentric rings on a red as opposed to a green background (Bedwell, Miller, Brown, \& Yanasak, 2006).

Notably, previous metacontrast visual backward masking studies have demonstrated a change in performance with a red background, consistent with suppression of the M pathway in healthy adults. In these studies, reduced masking was found with a red background as opposed to 
a white (Breitmeyer \& Williams, 1990; Edwards, Hogben, Clark, \& Pratt, 1996) or green (Breitmeyer, May, \& Heller, 1991; Breitmeyer \& Williams, 1990) background, as indicated by improved accuracy in identifying the target. Similarly, masking is reduced for isoluminant red masks as opposed to isoluminant green, blue, or white masks (Williams, Breitmeyer, Lovegrove, \& Gutierrez, 1991). These backward masking studies used "metacontrast" masking, in which the mask does not spatially overlap the target.

A more recent study examined the effect of a red background with a location backward masking task as opposed to that of a gray background (Bedwell, Brown, \& Miller, 2003). This study compared relatives of persons with schizophrenia with nonpsychiatric controls. Only the performance of the nonpsychiatric controls $(N=31)$ in that study is considered here. The targets were curved letter stimuli (, , C, O, or Q), and the mask was uppercase Xs that always appeared at all possible target locations, completely overlapping the targets. The straight lines of the Xs were not present in the curved target letters; thus, the mask did not share structural features with the target. As Breitmeyer (1984) defined masking, "when target and mask patterns share common structural features and overlap spatially, masking by structure prevails. A mask that consists of random elements with no obvious structural relation to features of the target pattern is employed in masking by noise" (p. 134; italics added). Although Bedwell et al.'s (2003) Xs mask did not have a random aspect to it - for example, a region of random dots - the complete lack of any structural similarities between their mask and targets appears more consistent with the definition of a noise mask. However, the term masking by noise is interpreted in various ways by different researchers, and some would consider this previous study to be masking by structure or pattern due to the lack of randomness in the mask.

They used SOAs of 26, 40, 53, and $93 \mathrm{msec}$, and required target localization (i.e., which of four possible quadrants on the screen) without having to actually identify the curved target letters. The color backgrounds in their study were set to physical equiluminance using a photometer to calibrate monitor luminance. Therefore, individual differences in basic wavelength sensitivity were not controlled for. Despite this uncontrolled error variance, the results revealed a main effect of color $\left(\eta^{2}=.37\right.$; large effect size $)$, with reduced accuracy in the red background condition. There was no statistical interaction between color and SOA; however, the 93-msec SOA showed the largest effect size for reduced accuracy to the red background.

This effect was interpreted to be largely due to the disruptive effect of the red background on target localization, which can be accomplished with information primarily via the $\mathrm{M}$ pathway. In backward masking paradigms requiring participants to simply indicate the target's location (and not identify it), information is provided by both the transient M pathway (used to initially detect the target's location) and the sustained signal from the P pathway (contributing further target information). The $\mathrm{M}$ and $\mathrm{P}$ pathway information coming from the target location then interacts with the transient $\mathrm{M}$ pathway signal from the mask(s). Although the mask would also elicit a P pathway signal, it is the earlier M pathway burst that is relevant to backward masking, since the observer is not asked to report on any sustained $(\mathrm{P})$ pathway detail about the mask. Although red light could have decreased the interfering effect of the mask (and therefore improved accuracy), the predominant effect of the red light on overall performance appears to be on disrupting initial target localization in this location masking by noise study. However, this interpretation is speculative, since the ability of diffuse red light to disrupt unmasked localization was not directly examined in that study, because unmasked trials were not included.

Skottun (2004) challenged the view that using red light in a psychophysical experiment infers effects of the attenuation of the M pathway in particular. As Skottun demonstrates, red light also substantially suppresses particular $\mathrm{P}$ cells $(+\mathrm{G}-\mathrm{R}$ and $+\mathrm{B}-\mathrm{Y})$ that are only modestly suppressed by green and blue filters. Therefore, although it has been clearly established that the M pathway is suppressed by red light, the particular performance differences in a given psychophysical task may be due to changes in the $\mathrm{M}$ and/or P pathway as a result of the red light (as opposed to other colors). An anonymous reviewer also proposed that red light may theoretically have a weaker potential for directed arousal than would green light. If so, red light may decrease the speed of processing for both the target and mask. Depending on the SOA and structural similarities between them, this reduced processing speed may result in either improved target detection (i.e., weaker masking) or reduced target detection (i.e., delayed processing of target). Although the exact nature and rationale of this arousal hypothesis remains unclear, it is possible that future experiments could be devised to test such a notion. Therefore, in light of the concerns raised by Skottun, it may be most appropriate to consider the use of red light as manipulating "relative" $\mathrm{M}$ activity. In our study, then, $M$ activity is expected to be relatively less in the red background condition than in the green.

In the present study, we explored the effect of a red background on location backward masking by using a mask that spatially overlaps the target and shares its primary structural features, which is referred to as backward masking by structure (Breitmeyer \& Ögmen, 2000). This type of backward masking is thought to be better able to reveal interactions between pathways at the cortical level (Breitmeyer \& Ögmen, 2000), similar to metacontrast masking studies that have shown increased accuracy (decreased masking) on red backgrounds. In comparison with Bedwell et al.'s (2003) masking study in which accuracy decreased on red backgrounds, we hypothesized that the present paradigm, which used a mask that shared primary structural features of the target, would lead to increased accuracy with a red background. Specifically, we theorized that a decreased $\mathrm{M}$ response to the mask would overcome potential decreases in target localization (decreasing accuracy) due to red light - the proposed mechanism for Bedwell et al.'s (2003) results using location masking by noise. The present study provides the first reported exploration of this redlight effect on a location backward masking by structure, using a paradigm in which the mask spatially overlaps the target and shares its primary structural features. 


\section{METHOD}

\section{Participants}

Adults from the community were recruited using newspaper advertisements for participation as nonpsychiatric controls in a larger psychiatric study. Exclusionary criteria included the presence of an Axis I psychiatric disorder (per clinical interview), psychotropic medication use (including narcotic pain medicine), corrected visual acuity of less than 20-30 (per Snellen wall chart), history of neurological symptoms, head trauma, and recent substance abuse.

Following the exclusionary criteria, data from 18 nonpsychiatric participants ( 9 female) were examined. The ages of the 18 participants ranged from 25 to 65 years $(M=45.9, S D=11.4)$. All participants completed the computerized visual backward masking task as part of a larger battery of testing.

\section{Stimuli and Apparatus}

The backward masking task was presented on a 22-in. CRT monitor (Iiyama Vision Master Pro 514), running at a $150-\mathrm{Hz}$ refresh rate. E-Prime software (Psychology Software Tools) was used to build and run the backward masking task. Participants were placed in a forehead-chinrest in order to minimize movement during the task. Participants were seated 18 in. from the monitor. The graphic images of the stimuli were provided by the UCLA group, and have been used in several recent schizophrenia studies out of that lab (Green, Mintz, et al., 2003; Green, Nuechterlein, \& Breitmeyer, 2002; Green, Nuechterlein, Breitmeyer, Tsuang, \& Mintz, 2003; Rassovsky, Green, Nuechterlein, Breitmeyer, \& Mintz, 2004).

\section{Procedure}

During each trial, a fixation symbol (a small black cross) was presented in the center of the screen for $300 \mathrm{msec}$, followed by a blank screen for $100 \mathrm{msec}$. A target then appeared for $13 \mathrm{msec}$ as a small black square (subtending a visual angle of $0.75^{\circ}$ ) with a small gap in one side (see Figure 1). The gap could appear at the top, bottom, or left side. This gap was irrelevant to the study, but it was present because it has been used in previous studies in which participants reported its location. Targets could appear at any one of four corners from the center of the screen (upper left, upper right, lower left, lower right) at a distance of $2.51^{\circ}$ from the center screen. The target was followed by a blank screen interstimulus interval (ISI) that varied randomly from a predefined list of possible values, as described below. The mask then appeared for $27 \mathrm{msec}$ (higher-than-target energy mask - twice the duration of target). The mask consisted of clusters of small squares that spatially overlapped the initial target (see Figure 1). In the case of the 0-msec SOA, the mask appeared simultaneously with the target.

Participants were asked to orally state the number of the spatial quadrant in which the target appeared. An oral response was chosen to reduce participant keypress errors and related attentional distraction. The quadrants were numbered (1-4), with numbers written in corresponding locations displayed on a label located on the top rim
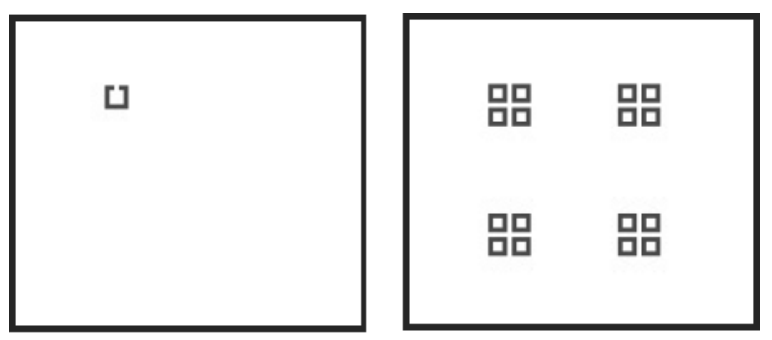

Target Mask

Figure 1. Example stimuli from the backward masking task. of the monitor so that it was not necessary for the participants to memorize the sequence. The trials were self-paced, since each subsequent trial was triggered when the investigator entered the participant's oral response approximately $2 \mathrm{sec}$ after the participant stated the answer.

SOA is defined as being the duration from the onset of the target to the onset of the mask. Thus, this includes the duration of the target $(13 \mathrm{msec})$ and the duration of the blank screen ISI. Nine SOAs were used $(0,27,33,40,47,54,60,74$, and $87 \mathrm{msec})$, with 12 trials presented at each SOA. In addition, 12 trials in which there was no mask following the target were included as a baseline measure of attention during the task. Thus, 120 stimuli were presented, with each stimulus chosen randomly from the entire list of possible stimuli and SOA combinations. Within each SOA and unmasked condition, the target appeared in each of the four locations at an equal frequency.

This entire task was first presented on either a red or a green background (counterbalanced over participants), followed by a 1-min rest, followed by the same task condition on the opposite color background (green or red). The two colored backgrounds were approximately matched for physical luminance $\left(29.3 \mathrm{~cd} / \mathrm{m}^{2}\right)$, as measured by a spot photometer placed near the middle of the screen. Prior to each condition, participants had a practice session that included 10 trials with no mask, followed by 10 trials with a long SOA $(132 \mathrm{msec})$ and a mask. None of the participants displayed any difficulty on the practice trials.

\section{RESULTS}

Accuracy was first examined for the 24 unmasked trials that were randomly interspersed throughout the task (averaged over green and red conditions). All participants achieved at least $84 \%$ accuracy on these unmasked trials (range, $84 \%$ to $100 \%, M=96.14 \%, S D=5.58 \%$ ), suggesting that all participants maintained adequate attention throughout the task. The accuracy on the unmasked trials did not statistically differ between background colors (using a Wilcoxon signed ranks test on the skewed distribution, $Z=1.47, p>.14$ ).

A two-factor repeated measures ANOVA was used to examine the interaction between color (two levels) and SOA (nine levels). The main effect of SOA was statistically significant $\left[F(8,136)=26.00, p<.001, \eta^{2}=.61\right]$. There was no suggestion of a nonlinear trend for SOA. The main effect of color background was also statistically significant $\left[F(1,136)=4.93, p<.04, \eta^{2}=.23\right.$; medium effect size; see Figure 2]. The interaction of color and SOA did not approach statistical significance $[F(8,136)=$ $1.09, p>$.36]. Exploratory post hoc analyses were conducted within each SOA using paired $t$ tests to determine the SOA(s) at which the change-to-color background was greatest. Results indicated that the most statistically significant increase in accuracy with the red (as opposed to the green) background occurred at the $47-\mathrm{msec}$ SOA $[t(17)=2.26, p<.04]$, followed by the 60 -msec SOA $[t(17)=2.07, p<.05$; see Figure 2]. Accuracy change by color did not approach statistical significance $(p>.10)$ with the remaining seven SOAs.

\section{DISCUSSION}

Consistent with our hypothesis, the color background did have an effect on masking performance with the red background, resulting in increased target localization ac- 


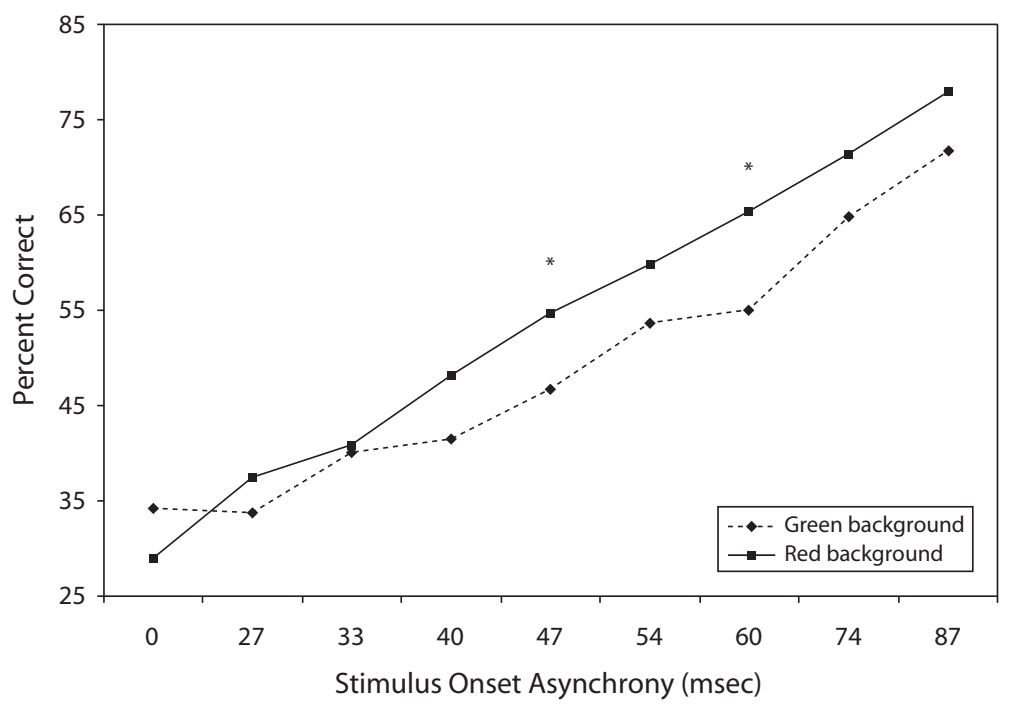

Figure 2. Backward masking accuracy by color background. ${ }^{*} p<.05$.

curacy (reduced masking) in comparison with that of the green background. The mechanism for this color effect is theorized to be due to red light suppressing the M pathway response to the mask, resulting in improved accuracy (i.e., increased target visibility).

These results are consistent with findings from earlier metacontrast studies reporting reduced masking with a red background. The present results support the premise that both metacontrast masking and pattern masking by structure are sensitive to subtle reductions in the strength of the $\mathrm{M}$ pathway response to the mask under red-light conditions. The present reduction in masking with red light is in the opposite direction from findings reported in an earlier masking-by-noise paradigm with a red background by Bedwell et al. (2003). Therefore, it is theorized that the finding from the earlier study of a decrease in accuracy being the result of a red background was primarily due to a reduction in the ability of the M pathway to contribute to target localization, with relatively little influence from a reduction in the strength of the mask. In contrast, it appears that in location masking by pattern, the reduction in the strength of the mask by red light outweighs the potential reduction in the ability to initially locate the target.

Although it is possible that the limit on performance in the paradigms masking potential target locations was due to basic target visibility rather than to localization per se, either possibility would rely on relatively more $\mathrm{M}$ than $\mathrm{P}$ pathway input, since target detail is not needed in either case (e.g., only the quadrant of the screen in which the target appeared is queried). If a strong $\mathrm{M}$ response to the mask is integral to producing masking, then the masking function from the present study indicates that as the timing between the target and mask increases, the mask becomes less effective. One theory is that as increasing SOA is reducing mask effectiveness, the effect of the red background emerges (at SOAs over $33 \mathrm{msec}$; see Figure 2) as a relatively greater $\mathrm{M}$ response to the mask on the green background. When overall
$\mathrm{M}$ activity is reduced on the red background, the mask has an additional loss of effectiveness. Using an alternate conceptualization, if the effect of the mask is considered as creating recurrent activity at the target location (Enns \& Di Lollo, 2000), at short SOAs, recurrent activity associated with the target is interfered with/negated by feedforward activity from the mask. At short SOAs, this interference appears to occur whether or not overall $\mathrm{M}$ activity is reduced by red light. Later, when mask recurrent activity is losing effectiveness (target processing is more complete), reducing $\mathrm{M}$ activity appears to make mask feedforward activity even less disruptive to target processing.

The earlier metacontrast studies did not involve locating a target, but instead involved identifying the detail of the target (a task requiring relatively more P pathway input). Therefore, the consistent findings of the metacontrast studies regarding the effect of a red background in decreasing the masking of the target likely reflect the relatively minor input required from the $\mathrm{M}$ pathway in the initial target identification in those studies (thus resulting in the consistent influence of red light in decreasing the M pathway response to the mask).

A potential limitation of the present study is the fixed background luminance (colors matched for physical luminance), which does not account for individual differences in contrast sensitivity to these colors. However, an earlier metacontrast study reported a similar attenuation of masking due to a red background, regardless of whether the backgrounds were set at a fixed physical or individualized psychophysical isoluminance (Breitmeyer \& Williams, 1990). Similarly, another previous metacontrast study used only fixed physical isoluminance between background colors (similar to this study) and reported a statistically significant effect of a red background in reducing masking as opposed to a white background (Edwards et al., 1996). Therefore, the present results are consistent with at least two other metacontrast studies reporting at- 
tenuated masking effects (increased accuracy) with a red background in comparison with other physically (not psychophysically) isoluminant background colors.

There was considerable individual variability in masking response to the red background. For example, at the most statistically significant SOA $(47 \mathrm{msec})$, the mean increase in accuracy to a red background was $7.89 \%(S D=14.82$, range $=-16$ to +33$)$. Although this may be related to individual differences in contrast sensitivity to the different color backgrounds, the fact that masking performance change to the red background did not differ when this was controlled for in a previous study (Breitmeyer \& Williams, 1990) suggests that individual variability may primarily be due to individual differences in $\mathrm{M}$ pathway suppression from red light. However, it is also possible that individual differences in the suppression of various $P$ pathway cells to red light may add to this variance (Skottun, 2004).

The present results provide the first report of evidence for the effect of red light to attenuate masking in a location backward masking by structure task. However, the present study cannot directly address whether this red-light effect is primarily due to M pathway suppression. Further research using event-related potentials or magnetoencephalography may be able to clarify the $\mathrm{M}$ and $\mathrm{P}$ pathway changes to red light that account for performance changes on particular psychophysical tasks. Further clarification of this red-light effect in nonpsychiatric adults is particularly important, since recent research has shown that the presence and/or direction of visual processing change to diffuse red light appears to be sensitive to genetic liability to schizophrenia (Bedwell et al., 2003; Bedwell et al., 2006).

\section{AUTHOR NOTE}

The authors thank Bruno Breitmeyer for help with the interpretation of the findings. In addition, the authors thank Vidya Kamath for help in recruiting the participants, Reesa Donnelly for help in collecting data, Adam McCowan for data entry, and the Center for Behavioral Health at Florida Hospital Orlando for providing the laboratory space used for data collection. Correspondence concerning this article should be addressed to J. S. Bedwell, Department of Psychology, 4000 Central Florida Blvd., University of Central Florida, Orlando, FL 32816-1390 (e-mail: jbedwell@mail.ucf.edu).

\section{REFERENCES}

Bedwell, J. S., Brown, J. M., \& Miller, L. S. (2003). The magnocellular visual system and schizophrenia: What can the color red tell us? Schizophrenia Research, 63, 273-284.

Bedwell, J. S., Miller, L. S., Brown, J. M., \& Yanasak, N. E. (2006). Schizophrenia and red light: fMRI evidence for a novel biobehavioral marker. International Journal of Neuroscience, 116, 881-894.

Breitmeyer, B. G. (1984). Visual masking: An integrative approach. New York: Oxford University Press.

Breitmeyer, B. G., \& Breier, J. I. (1994). Effects of background color on reaction time to stimuli varying in size and contrast: Inferences about human M channels. Vision Research, 34, 1039-1045.

Breitmeyer, B. G., \& GanZ, L. (1976). Implications of sustained and transient channels for theories of visual pattern masking, saccadic suppression, and information processing. Psychological Review, 83, 1-36.

Breitmeyer, B. G., May, J. G., \& Heller, S. S. (1991). Metacontrast reveals asymmetries at red-green isoluminance. Journal of the Optical Society of America A, 8, 1324-1329.

Breitmeyer, B. G., \& Ögmen, H. (2000). Recent models and findings in visual backward masking: A comparison, review, and update. Perception \& Psychophysics, 62, 1572-1595.

Breitmeyer, B. G., \& Williams, M. C. (1990). Effects of isoluminantbackground color on metacontrast and stroboscopic motion: Interactions between sustained $(\mathrm{P})$ and transient $(\mathrm{M})$ channels. Vision Research, 30, 1069-1075.

Brown, J. M., \& Косн, C. (2000). Influences of occlusion, color, and luminance on the perception of fragmented pictures. Perception \& Motor Skills, 90, 1033-1044.

Chapman, C., Hoag, R., \& Giaschi, D. (2004). The effect of disrupting the human magnocellular pathway on global motion perception. Vision Research, 44, 2551-2557.

Chase, C., Ashourzadeh, A., Kelly, C., Monfette, S., \& Kinsey, K. (2003). Can the magnocellular pathway read? Evidence from studies of color. Vision Research, 43, 1211-1222.

DE Monasterio, F. M. (1978). Properties of concentrically organized X and Y ganglion cells of macaque retina. Journal of Neurophysiology, 41, 1394-1417.

Dreher, B., FuKada, Y., \& Rodieck, R. W. (1976). Identification, classification and anatomical segregation of cells with X-like and Y-like properties in the lateral geniculate nucleus of old-world primates. Journal of Physiology, 258, 433-452.

Edwards, V. T., Hogben, J. H., Clark, C. D., \& Pratt, C. (1996). Effects of a red background on magnocellular functioning in average and specifically disabled readers. Vision Research, 36, 1037-1045.

EnNS, J. T., \& Di Lollo, V. (2000). What's new in visual masking? Trends in Cognitive Sciences, 4, 345-352.

Green, M. F., Mintz, J., Salveson, D., Nuechterlein, K. H., BreitMEYER, B., Light, G. A., \& BrafF, D. L. (2003). Visual masking as a probe for abnormal gamma range activity in schizophrenia. Biological Psychiatry, 53, 1113-1119.

Green, M. F., Nuechterlein, K. H., \& Breitmeyer, B. (2002). Development of a computerized assessment for visual masking. International Journal of Methods in Psychiatric Research, 11, 83-89.

Green, M. F., Nuechterlein, K. H., Breitmeyer, B., Tsuang, J., \& MinTz, J. (2003). Forward and backward visual masking in schizophrenia: Influence of age. Psychological Medicine, 33, 887-895.

KRÜGER, J. (1977). Stimulus dependent colour specificity of monkey lateral geniculate neurones. Experimental Brain Research, 30, 297-311.

Livingstone, M. S., \& Hubel, D. H. (1984). Anatomy and physiology of a color system in the primate visual cortex. Journal of Neuroscience, 4, 309-356.

Livingstone, M. S., \& Hubel, D. H. (1987). Psychophysical evidence for separate channels for the perception of form, color, movement, and depth. Journal of Neuroscience, 7, 3416-3468.

Purushothaman, G., Ögmen, H., \& Bedell, H. E. (2000). Gammarange oscillations in backward-masking functions and their putative neural correlates. Psychological Review, 107, 556-577.

Rassovsky, Y., Green, M. F., Nuechterlein, K. H., Breitmeyer, B., \& MinTz, J. (2004). Paracontrast and metacontrast in schizophrenia: Clarifying the mechanism for visual masking deficits. Schizophrenia Research, 71, 485-492.

Skotтun, B. C. (2004). On the use of red stimuli to isolate magnocellular responses in psychophysical experiments: A perspective. Visual Neuroscience, 21, 63-68.

Wiesel, T. N., \& Hubel, D. H. (1966). Spatial and chromatic interactions in the lateral geniculate nucleus body of the rhesus monkey. Journal of Neurophysiology, 29, 1115-1156.

Williams, M. C., Breitmeyer, B. G., Lovegrove, W. J., \& GutierREZ, C. (1991). Metacontrast with masks varying in spatial frequency and wavelength. Vision Research, 31, 2017-2023.

(Manuscript received November 6, 2006; revision accepted for publication November 5, 2007.) 